\title{
Assessment of COVID-19 pandemic stress among health sciences students in a private university in South-West Nigeria
}

\author{
Peter Oluyemi Adedeji ${ }^{*}$, Aarinola Precious Oyedeji ${ }^{1}$, Adenike Ayobola Olaogun ${ }^{1}$, Oluwatosin \\ Adewusi Adedeji
}

\begin{abstract}
Background: COVID-19 pandemic has been reported to have psychological effects on various aspects of human life and segments of society. The study aimed to assess COVID-19 pandemic stress during and after lockdown among health sciences students at a private university in South-West, Nigeria.

Method: A descriptive, cross-sectional web-based research was conducted in a private teaching hospital of a private university using a simple random sampling technique. The study instrument was a self-structured questionnaire containing sociodemographic details and questions adapted from the validated Perceived Stress Scale (PSS) by Sheldon Cohen regarding lectures during and after COVID-19 lockdown. Data were analyzed with the aid of SPSS version 26 software.
\end{abstract}

Results: A total of 220 respondents were included in the study, with a mean age of $22.08 \pm 2.996$. Most of the respondents were females $(157,71.4 \%)$ in their 4th-year level $(37 \%)$. The participants had a high level of stress during (46.8\%) COVID-19 lockdown than after the lockdown (29.1\%), and statistical significance was observed between the perceived stress level during and after the COVID-19 lockdown (McNemar-Bowker's value $=29.322, P=<0.001)$ at $P<0.05$. The female gender $(B=2.432, P=0.03)$ and the respondents in 3rd year $(B=-4.178, P=0.035)$ had moderate stress during COVID-19 lockdown at $P<0.05$. After the COVID-19 lockdown, the respondents in their 5th year had both moderate $(B=-1.73, P=0.022)$ and high $(B=-2.08, P=0.026)$ stress at $p<0.05$ respectively. The respondents of age groups $17-20$ years $(B=3.28, P=0.04)$ and $21-24$ years $(B=2.93, P=0.046)$ also had high stress at $P<0.05$ after the lockdown. Most respondents suggested reducing online lecture loads and lockdown in school as ways the university can help students cope with stress in case of repeat pandemic outbreaks in the future.

Conclusion: This study concludes that the health science students experienced a high level of stress during the COVID-19 lockdown and moderate stress level after the lockdown was eased.

Keywords: COVID-19 Pandemic, Online Classes, Perceived Stress Level, Private University, Health Sciences Students, Lockdown, Nigeria

\section{Background}

The 2019 corona virus disease (COVID-19), a highly infectious disease caused by severe acute respiratory syndrome Coronavirus 2 (SARS-CoV-2), was first reported in Wuhan, Hubei province, China in December 2019 [1,2]. It was declared an international public health emergency on January 30, 2020, by the World Health Organization (WHO) and has been said to have affected over 210 countries, including Nigeria [3,4]. There

*Correspondence: peteradedeji2020@gmail.com

${ }^{1}$ Department of Nursing Science, College of Health Sciences, Bowen University, Iwo, Osun State, Nigeria.

Full list of author information is available at the end of the article have been about 40 million confirmed cases of COVID-19, with 1.1 million deaths across the world as of October 18, 2020 [4]. Having declared COVID-19 a global pandemic, many countries, including Nigeria, came up with strict precautions on their citizens in an attempt to control the spread; these include the closure of schools and other public places of aggregation, termination of public gatherings, imposition of travel bans and curfews, and compulsory quarantine of people who have come into contact with the infected people [5-9]. The pandemic and the measures taken to halt its spread stressed people from all walks of life, and the students were not exempt [10,11]. Relatively high rates of anxiety, depression, post-traumatic 
stress disorder, psychological distress, and stress were reported in the general population during the COVID-19 pandemic in eight countries [12]. The stress levels of general populations, including office employees, daily wagers, health care providers, and the students (especially the health sciences students), have indeed been elevated due to the present pandemic, as is evident from various local and international studies which have been carried out to assess the mental impact and study the related diverse factors of the COVID-19 pandemic [13].

Most public tertiary education institutions opted for a complete shutdown of all learning activities, but the private ones opted for virtual/online learning to engage their students during the pandemic while in the confine of their homes. In a short period, these students at private universities, especially health sciences students, had to adjust to new living circumstances and adapt to online learning platforms, particularly in courses that require high levels of interaction and hands-on experiences such as practicums and laboratory performance that were not initially designed for online delivery. They face many difficulties because they depend solely on gaining their learning experience both in the lecture theatres and at the bedside; this has been severely compromised due to the closure of the medical schools [13]. The sudden isolation and social distancing are projected to significantly affect the mental health of these health sciences students as they are removed from their extracurricular activities, feel less connected with their friends, hobbies and uncertain about their future, their health, and that of their friends and relatives [16]. Misinformation about the pandemic circulating online and social media may also contribute immensely to stress among these students and their friends [17-19].

The response of health care students to such critical situations may vary from self-contained anxiety to suicidal attempts [14]. Reduced social interaction and interpersonal relationships may also occur as a result of loneliness [15]. While several studies have reported a high level of academic stress among students receiving face-to-face class lectures [20], there is a need to assess the likelihood of COVID-19 pandemic stress on students at private universities that continued learning activities during the total lockdown. This study assessed the level of perceived stress during and after the COVID-19 lockdown among Health Sciences students of a private teaching hospital, Iwo, Nigeria.

\section{Method}

Design and setting

A web-based descriptive, cross-sectional research design was used to assess COVID-19 Pandemic Stress during and after lockdown among Health Sciences Students of Bowen University, Iwo at Bowen University Teaching Hospital, the teaching hospital of Bowen University, located at Ogbomosho, Nigeria. The teaching hospital has over 400 bed capacity with a multidisciplinary facility, over 800 staff and students' facility, 50,000 outpatients, and 10,000 patients with specialties in family medicine, community health, accident and emergency, nursing services, intensive care, pediatrics, general surgery, dentistry, anatomical pathology among others.

\section{Target population}

A total of 412 undergraduate health sciences students studying in the teaching hospital were in the 3rd year (300 level) to 6th year (600 level) at the time of the study. The population comprised 227 medical students, 132 nursing students, and 53 physiotherapy students.

\section{Sample size}

The sample size was calculated using Taro Yamene (1967) formula; $(\mathrm{n})=\mathrm{N} \div[1+\mathrm{N}(\mathrm{e} 2)] ; \mathrm{N}=412$ and $\mathrm{e}=0.05$ (assumed error). The final sample size was calculated to 220 after adding $10 \%$ non-response rate.

\section{Sampling techniques}

A simple random sampling method was used in this study. The students were all informed about the study on their various WhatsApp groups through their class representatives and group administrators. Those interested in participating in the study were asked to send private WhatsApp messages to the researcher. All those who consented were contacted to be added to another WhatsApp group with more details about the study.

\section{Inclusion and Exclusion Criteria}

Undergraduate's students of 3rd, 4th, 5th and 6th batches, both gender of nursing, medicine and physiotherapy in the College of Health Sciences of Bowen University who had spent at least one semester at Bowen University Teaching hospital before the COVID-19 pandemic lockdown who were willing to participate in the study were included. In contrast, those who were not willing to participate were excluded. Also, the medical and physiotherapy students posted at Ejigbo, the annex of the teaching hospital, were excluded.

The instrument for Data collection

The research instrument used for data collection was a selfstructured questionnaire containing the following four sections:

Section A: Sociodemographic data: containing 10 Items which include age, gender, department, year in school, religion, monthly allowance in school, monthly allowance during COVID-19 lockdown.

Section B and C: Perceived Stress Scale (PSS): A standardized instrument developed by Sheldon Cohen (1983). The PSS consists of 10 questions, each adapted to assess the level of perceived stress during and after COVID-19 lockdown among respondents in this study.

Section D: This is a blank space for the respondents to fill in their recommendations to the University management on how to help cope with possible stress in case of another future pandemic or similar COVID-19 lockdown.

\section{Validity and Reliability of Instrument}

The questionnaire was written in English language and was read through and approved by the experts from the nursing departmental board of Bowen University to ensure clarity and easy comprehension. A pilot study was also used to validate that the questionnaire was tested among 30 medical and nursing students (not included in the study). The Cronbach's alpha was used to assess the reliability of the questionnaire and was found to be 0.86 .

\section{Dependent variable}

The Perceived Stress Scale (PSS) consists of 10 questions, each adapted to assess the level of perceived stress during and after 
COVID-19 lockdown among respondents in this study. The respondents were asked how often they had felt a particular sensation during and after the lockdown. A 5-point Likert scale (ranging from $0=$ "never" to $4=$ "very often") was used to grade the levels of perceived stress. The global PSS score ranges from 0 to 40 , with higher scores indicating higher levels of perceived stress. A score ranging from $0-13$ would be considered low stress; a score between 14 and 26 represents moderate stress. A score ranging from 27-40 represents a high level of perceived stress. There is a norm table for the PSS-10 item inventory".

\section{Independent variables}

The age of the participants is categorized as "17-20 years", "2124 years", "25-28 years," and "29-32 years". Their gender is recorded as "Male" and "Female". The religion of participants has been categorized into "Christianity" and "Islam". The department of participants which refers to their course of study was categorized as "Medicine", "Nursing" and "Physiotherapy". The year of study of the respondents referred to as levels in this study was categorized as "3rd year ", "4th year ", "5th year," and "6th year". The monthly allowance of the respondents before COVID-19 lockdown was classified as "5000-15000", "16000-30000", "31000-50000", and " 51000-80000" in naira. During the COVID-19 lockdown, the monthly allowance of the participants was categorized in naira as " Zero (0)", "200-4000", "4100-15000", "16000-40000", "41000-60000" and " 6100080000". Where the participants lived during the COVID-19 lockdown is categorized as "Alone", "With friends", "With parents", "With relatives," and "With sister". How often the participants read news online about COVID-19 is categorized as "Always", "Sometimes", "Rarely", and "Never". The platform with which the respondents received online lectures during the COVID-19 lockdown was categorized as "WhatsApp", "Zoom", "Zoom, Google meet", " Zoom, Google meet, WhatsApp", " Zoom, WhatsApp", " Zoom, Google meet, Google classroom" and " Telegram".

\section{Statistical analysis}

Data collected were analyzed using the IBM Statistical Product and Service Solutions (SPSS) Windows version 26. Descriptive statistics were presented in tables as mean, frequencies, and percentages. Inferential statistics including multivariate logistic regression to ascertain the relationship between the sociodemographic factors and the perceived stress scale (PSS) during and after COVID-19 lockdown. In contrast, McNemarBowker was used to test the difference between the perceived stress before and after COVID-19 lockdown was carried out using statistical significance at a p-value less than 0.05 .

\section{Results}

Sociodemographic characteristics

A total of 220 respondents were included in the study, with a mean age of $22.08 \pm 2.996$ (mean $\pm \mathrm{SD}$ ) ranging from the lowest age of 17 years to the highest age of 32 years (Table 1). Most of the respondents were females $(157,71.4 \%)$, in their 4th year (37\%) who lived with their parents during the COVID19lockdown (88.2\%), received 21,000 to 30,000 naira (31.8\%) as monthly allowance before COVID-19 lockdown but nothing (37.3\%) during the lockdown, and received their online lectures via $(92,41.8 \%)$ (Table1).
Table 1: Sociodemographic data of respondents $(n=220)$

\begin{tabular}{|c|c|}
\hline Variables & $\mathbf{N}(\%)$ \\
\hline \multicolumn{2}{|l|}{$\begin{array}{l}\text { Age (Mean Age } \pm \text { SD }=22.08 \pm 2.996, \text { Min }=17 \\
\operatorname{Max}=32 \text { ) }\end{array}$} \\
\hline 17-20years & $82(37.3)$ \\
\hline 21-24years & $94(42.7)$ \\
\hline 25-28years & $34(15.5)$ \\
\hline 29-32years & $10(4.5)$ \\
\hline \multicolumn{2}{|l|}{ Gender } \\
\hline Male & $63(28.6)$ \\
\hline Female & $157(71.4)$ \\
\hline \multicolumn{2}{|l|}{ Department } \\
\hline Medicine & $82(37.3)$ \\
\hline Nursing & $95(43.2)$ \\
\hline Physiotherapy & $43(19.5)$ \\
\hline \multicolumn{2}{|l|}{ Year in School } \\
\hline $3^{\text {rd }}$ & $42(19.1)$ \\
\hline $4^{\text {th }}$ & $77(35)$ \\
\hline $5^{\text {th }}$ & $70(31.8)$ \\
\hline $6^{\text {th }}$ & $31(14.1)$ \\
\hline \multicolumn{2}{|l|}{ Religion } \\
\hline Christianity & $214(97.3)$ \\
\hline Islam & $6(2.7)$ \\
\hline \multicolumn{2}{|l|}{ Monthly allowance in school } \\
\hline $5000-15000$ & $18(8.2)$ \\
\hline $16000-30000$ & $110(50)$ \\
\hline $31000-50000$ & $72(32.7)$ \\
\hline $51000-80000$ & $20(9.1)$ \\
\hline \multicolumn{2}{|l|}{$\begin{array}{llll}\begin{array}{l}\text { Monthly } \\
\text { lockdown }\end{array} & \text { allowance } & \text { during } & \text { COVID-19 } \\
\end{array}$} \\
\hline Zero $(0)$ & $83(37.7)$ \\
\hline $200-4000$ & $22(10)$ \\
\hline $4100-15000$ & $80(58.4)$ \\
\hline $16000-40000$ & $28(12.7)$ \\
\hline $41000-60000$ & $5(2.3)$ \\
\hline $61000-80000$ & $2(0.9)$ \\
\hline \multicolumn{2}{|l|}{$\begin{array}{l}\text { Where did you live during the COVID-19 } \\
\text { period }\end{array}$} \\
\hline Alone & $2(0.9)$ \\
\hline With friends & $9(4.1)$ \\
\hline With parents & $194(88.2)$ \\
\hline With relatives & $13(5.9)$ \\
\hline With sister & $2(0.9)$ \\
\hline \multicolumn{2}{|l|}{$\begin{array}{l}\text { How Often did you read about COVID-19 } \\
\text { news online during the lockdown? }\end{array}$} \\
\hline Always & $97(44.1)$ \\
\hline Sometimes & $93(42.3)$ \\
\hline Rarely & $26(11.8)$ \\
\hline Never & $4(1.8)$ \\
\hline \multicolumn{2}{|l|}{$\begin{array}{l}\text { Which platform was used for an online lecture } \\
\text { during the lockdown }\end{array}$} \\
\hline WhatsApp & $11(5)$ \\
\hline Zoom & $92(41.8)$ \\
\hline Zoom, Google meet & $8(3.6)$ \\
\hline Zoom, Google meet, WhatsApp & $61(27.7)$ \\
\hline Zoom, WhatsApp & $42(19.1)$ \\
\hline Zoom, WhatsApp, Google classroom & $4(1.9)$ \\
\hline Telegram & $2(1)$ \\
\hline
\end{tabular}


Perceived stress among health sciences students during the COVID-19 lockdown and after COVID-19 resumption The results showed that during the lockdown, been upset because of the unexpected changes in studies from face-to-face to online (Mean, $\mathrm{SD}=3.10,0.87$ ) ranked the highest level of perceived stress during COVID-19 among the health sciences students at Bowen University (Table 2). Table 3 shows that after COVID-19 resumption, the highest perceived stress was: how often they were able to control irritations in their life (Mean, $\mathrm{SD}=2.34,1.04$ ); how often they felt confident about their ability to handle various personal problems and challenges (Mean, SD $=2.30,1.05$ ); and how often the students felt that they could not cope with all the things that they had to do (Mean, SD $=2.30,1.05$ ), respectively. Of the respondents, $34.5 \%$ sometimes was able to control irritations in their life, $39.1 \%$ felt confident about their ability to handle various personal problems and challenges after COVID-19 resumption, while $22.3 \%$ were angered because of things they could not control as it relates to the pandemic and their studies.

Table 2: Perceived stress among health sciences students during the COVID-19 lockdown ( $\mathrm{n}=220)$

\begin{tabular}{|l|l|l|l|l|l|l|}
\hline Variables & $\begin{array}{l}\text { Mean } \\
\text { (SD) }\end{array}$ & $\begin{array}{l}\text { Very } \\
\text { Often } \\
\mathbf{n}(\%)\end{array}$ & $\begin{array}{l}\text { Often } \\
\mathbf{n}(\%)\end{array}$ & $\begin{array}{l}\text { Sometimes } \\
\mathbf{n}(\%)\end{array}$ & $\begin{array}{l}\text { Rarely } \\
\mathbf{n}(\%)\end{array}$ & $\begin{array}{l}\text { Never } \\
\mathbf{n}(\%)\end{array}$ \\
\hline $\begin{array}{l}\text { During the COVID-19 Lockdown, how often did you feel upset } \\
\text { because of unexpected changes in your studies from face to } \\
\text { face to online }\end{array}$ & $3.10(0.87)$ & $85(38.6)$ & $80(36.4)$ & $47(21.4)$ & $7(3.2)$ & $1(0.5)$ \\
\hline $\begin{array}{l}\text { During the COVID-19 Lockdown, how often did you feel that } \\
\text { you were unable to control the important things in your life }\end{array}$ & $2.77(0.99)$ & $57(25.9)$ & $83(37.7)$ & $56(25.5)$ & $20(9.1)$ & $4(1.8)$ \\
\hline $\begin{array}{l}\text { During the COVID-19 Lockdown, how often did you feel } \\
\text { nervous and "stressed." }\end{array}$ & $2.83(0.98)$ & $64(29.1)$ & $75(34.1)$ & $64(29.1)$ & $13(5.9)$ & $4(1.8)$ \\
\hline $\begin{array}{l}\text { During the COVID-19 Lockdown, how often did you feel } \\
\text { confident about your ability to handle various personal } \\
\text { problems and challenges }\end{array}$ & $2.59(0.91)$ & $37(16.9)$ & $80(36.4)$ & $81(36.8)$ & $20(9.1)$ & $2(0.9)$ \\
\hline $\begin{array}{l}\text { During the COVID-19 Lockdown, how often did you feel that } \\
\text { things were going your way }\end{array}$ & $2.39(1.12)$ & $47(21.4)$ & $45(20.5)$ & $87(39.5)$ & $30(13.6)$ & $11(5)$ \\
\hline $\begin{array}{l}\text { During the COVID-19 Lockdown, how often did you feel that } \\
\text { you could not cope with all the things that you had to do }\end{array}$ & $2.61(1.04)$ & $49(22.3)$ & $73(33.2)$ & $68(30.9)$ & $23(10.5)$ & $7(3.2)$ \\
\hline $\begin{array}{l}\text { During the COVID-19 Lockdown, how often were you able to } \\
\text { control irritations in your life? }\end{array}$ & $2.50(1.01)$ & $44(20)$ & $59(26.8)$ & $84(38.2)$ & $30(13.6)$ & $3(1.4)$ \\
\hline $\begin{array}{l}\text { During the COVID-19 Lockdown, how often did you feel that } \\
\text { you were on top of things }\end{array}$ & $2.39(0.97)$ & $29(13.2)$ & $65(29.5)$ & $88(40)$ & $32(14.5)$ & $6(2.7)$ \\
\hline $\begin{array}{l}\text { During the COVID-19 Lockdown, how often were you angered } \\
\text { because of things you could not control }\end{array}$ & $2.80(1.03)$ & $67(30.5)$ & $67(30.5)$ & $65(29.5)$ & $16(7.3)$ & $5(2.3)$ \\
\hline
\end{tabular}

Table 3: Perceived Stress among Health Sciences Students after the COVID-19 resumption ( $\mathrm{n}=220)$

\begin{tabular}{|l|l|l|l|l|l|l|l|}
\hline Variables & $\begin{array}{l}\text { Mean } \\
\text { (SD) }\end{array}$ & $\begin{array}{l}\text { Very } \\
\text { Often } \\
(\%)\end{array}$ & $\begin{array}{l}\text { Often } \\
(\%)\end{array}$ & $\begin{array}{l}\text { Sometimes } \\
(\%)\end{array}$ & $\begin{array}{l}\text { Rarely } \\
(\%)\end{array}$ & $\begin{array}{l}\text { Never } \\
(\%)\end{array}$ \\
\hline $\begin{array}{l}\text { After COVID-19 resumption, how often did you feel upset } \\
\text { because of your studies that changed unexpectedly }\end{array}$ & $2.24(1.09)$ & $31(14.1)$ & $59(26.8)$ & $73(33.2)$ & $44(20.0)$ & $13(6.0)$ \\
\hline $\begin{array}{l}\text { After COVID-19 resumption, how often did you feel that you } \\
\text { were unable to control the important things in your life }\end{array}$ & $2.14(1.06)$ & $25(11.4)$ & $52(23.7)$ & $82(37.3)$ & $49(22.3)$ & $12(5.5)$ \\
\hline $\begin{array}{l}\text { After COVID-19 resumption, how often did you feel nervous } \\
\text { and "stressed." }\end{array}$ & $2.23(1.04)$ & $25(11.4)$ & $64(29.1)$ & $76(34.6)$ & $\begin{array}{l}45(20.5 \\
\%)\end{array}$ & $\begin{array}{l}10(4.5 \\
\%)\end{array}$ \\
\hline $\begin{array}{l}\text { After COVID-19 resumption, how often did you feel confident } \\
\text { about your ability to handle various personal problems and } \\
\text { challenges. }\end{array}$ & $2.30(1.05)$ & $30(13.6)$ & $61(27.7)$ & $31(14.1)$ & $86(39.1)$ & $12(5.5)$ \\
\hline $\begin{array}{l}\text { After COVID-19 resumption, how often did you feel that things } \\
\text { were going your way? }\end{array}$ & $2.24(1.00)$ & $24.9(10.9)$ & $\begin{array}{l}58(26.4 \\
\%)\end{array}$ & $96(42.7 \%)$ & $30(13.6)$ & $12(5.5)$ \\
\hline $\begin{array}{l}\text { After COVID-19 resumption, how often did you feel that you } \\
\text { could not cope with all the things that you had to do. }\end{array}$ & $2.30(1.05)$ & $21(9.5)$ & $50(22.7)$ & $82(37.3)$ & $50(22.7)$ & $17(7.7)$ \\
\hline $\begin{array}{l}\text { After COVID-19 resumption, how often were you able to } \\
\text { control irritations in your life. }\end{array}$ & $2.34(1.04)$ & $29(13.2)$ & $74(33.6)$ & $76(34.5)$ & $32(14.5)$ & $9(4.1)$ \\
\hline $\begin{array}{l}\text { After COVID-19 resumption, how often did you feel that you } \\
\text { were on top of things. }\end{array}$ & $2.28(1.04)$ & $29(13.2)$ & $57(25.9)$ & $89(40.4)$ & $33(15.0)$ & $12(5.5)$ \\
\hline $\begin{array}{l}\text { After COVID-19 resumption, how often were you angered } \\
\text { because of things you could not control. }\end{array}$ & $2.05(1.05)$ & $21(9.5)$ & $52(23.6)$ & $82(37.3)$ & $49(22.3)$ & $16(7.3)$ \\
\hline
\end{tabular}


Level of perceived stress during COVID-19 lockdown and after COVID-19 resumption among health sciences students The results of the level of perceived stress during and after covid-19 lockdown among health sciences students of Bowen University shows that the highest proportion of the respondents (50\%) perceived moderate stress, $46.8 \%$ perceived high stress, while $3.2 \%$ perceived low stress during the COVID-19 pandemic (Figure 1). A high proportion of the respondents had perceived moderate stress after the COVID-19 lockdown (58.2\%), while $12.7 \%$ had perceived low stress after the COVID-19 resumption (Figure 1).

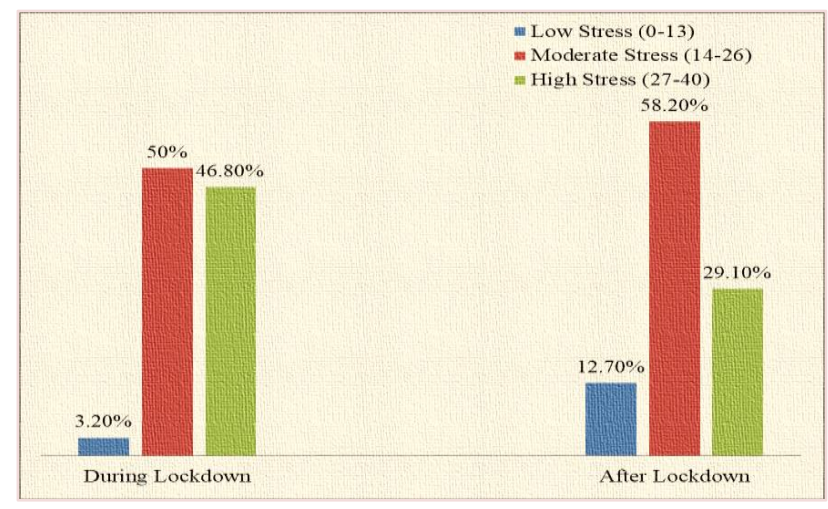

Figure 1: Level of Perceived Stress during COVID-19 Lockdown and after COVID-19 Resumption among Health Sciences Students
The result further showed that the participants had a high level of stress during $(46.8 \%)$ COVID-19 lockdown than after the lockdown (29.1\%). The McNemar-Bowker's test showed that there is a significant difference between the perceived stress level during and after the COVID-19 lockdown among the health sciences students at Bowen University (McNemarBowker's value $=29.322, \mathrm{p}=<0.001)$ at $\mathrm{p}<0.05$ (Table 4).

Relationships between some demographic factors and Perceived stress level during COVID-19 lockdown and after COVID-19 resumption

The result of multinomial regression of relationship between sociodemographic factors and perceived level of stress during covid-19 lockdown among the health sciences students of Bowen University (Table 5a) showed that the female gender (B $=2.432, \mathrm{p}=0.03)$ and the respondents in their 3rd year $(\mathrm{B}=-$ 4.178, $\mathrm{p}=0.035$ ) showed a statistical relationship with the moderate perceived stress during covid-19 lockdown at $\mathrm{p}<0.05$. After the COVID-19 lockdown, the results showed that the respondents in their 5 th year showed statistical relationship with both moderate $(\mathrm{B}=-1.73, \mathrm{p}=0.022)$ and high $(\mathrm{B}=-2.08, \mathrm{p}=$ 0.026 ) perceived stress at $p<0.05$ respectively (Table $5 b$ ). Also, the respondents of age groups 17-20 years $(B=3.28, p=0.04)$ and 21-24 years $(\mathrm{B}=2.93, \mathrm{p}=0.046)$ showed statistical relationship with high perceived stress at $\mathrm{p}<0.05$ after the lockdown (Table 5b).

Table 4: McNemar-Bowker's test of perceived stress scale during and after COVID-19 lockdown ( $\mathrm{n}=220)$

\begin{tabular}{|c|c|c|c|c|c|}
\hline & \multirow[t]{2}{*}{ Level of stress } & \multicolumn{3}{|c|}{ Perceived Stress Scale During Lockdown } & \multirow{2}{*}{$\begin{array}{l}\text { McNemar-Bowker Test; } \\
\text { df; p-value }\end{array}$} \\
\hline & & $\begin{array}{l}\text { Low Stress } \\
(0-13)\end{array}$ & $\begin{array}{l}\text { Moderate Stress } \\
(14-26)\end{array}$ & $\begin{array}{l}\text { High Stress } \\
(27-40)\end{array}$ & \\
\hline \multirow{3}{*}{$\begin{array}{l}\text { Perceived } \\
\text { Stress after } \\
\text { Lockdown }\end{array}$} & Low stress $(0-13)$ & $3(10.7)$ & $13(46.4)$ & $12(42.9)$ & \multirow{3}{*}{$\begin{array}{l}\text { McNemar-Bowker test }=29.322 \\
\mathrm{df}=3 \\
\text { p-value }=<0.001 *\end{array}$} \\
\hline & Moderate stress (14-26) & $1(0.8)$ & $79(61.7)$ & $48(37.5)$ & \\
\hline & High stress (17-40) & $3(4.7)$ & $18(28.1)$ & $43(67.2)$ & \\
\hline
\end{tabular}

Table 5a: Relationship between some demographic factors and perceived stress score during COVID-19 lockdown ( $\mathrm{n}=220)$

\begin{tabular}{|c|c|c|c|c|c|c|c|}
\hline Variables & Categories & B & SE & Wald & Sig. & $\operatorname{Exp}(B)$ & $\begin{array}{l}95 \% \mathrm{CI} \text { for } \operatorname{Exp}(\mathrm{B}) \\
\text { Lower-Upper }\end{array}$ \\
\hline & Moderate Stress (14-26) & & & & & & \\
\hline & Intercept & 1.95 & 2.02 & 0.93 & 0.33 & & \\
\hline \multirow[t]{4}{*}{ Age } & $17-20$ years & 3.26 & 2.07 & 2.49 & 0.12 & 26.08 & $0.45-1500.45$ \\
\hline & 21-24years & 1.58 & 1.67 & 0.90 & 0.34 & 4.88 & $0.19-128.25$ \\
\hline & 25-28years & 0.30 & 1.77 & 0.03 & 0.87 & 1.35 & $0.04-43.68$ \\
\hline & 29-32years & $0 \mathrm{~b}$ & & & & . & . \\
\hline \multirow[t]{2}{*}{ Gender } & Female & 2.43 & 1.12 & 4.73 & $0.03 *$ & 11.38 & $1.27-101.95$ \\
\hline & Male & $0 \mathrm{~b}$ & & & & & $\therefore$. \\
\hline \multirow[t]{3}{*}{ Department } & Medicine & -0.22 & 1.56 & 0.02 & 0.88 & 0.80 & $0.04-16.77$ \\
\hline & Nursing & -0.28 & 1.41 & 0.04 & 0.84 & 0.75 & $0.05-12.13$ \\
\hline & Physiotherapy & $0 \mathrm{~b}$ & & & & &.- \\
\hline \multirow[t]{6}{*}{ Level } & $3 \mathrm{rd}$ & -4.18 & 1.98 & 4.46 & $0.04 *$ & 0.02 & $0.0-0.74$ \\
\hline & 4th & -2.16 & 1.62 & 1.78 & 0.18 & 0.12 & $0.005-2.76$ \\
\hline & 5th & -0.89 & 1.73 & 0.27 & 0.61 & 0.41 & $0.01-12.04$ \\
\hline & 6th & $0 \mathrm{~b}$ & 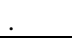 & . & . &. &. \\
\hline & High Stress (27-40) & & & & & & \\
\hline & Intercept & 1.86 & 2 & 0.86 & 0.35 & & \\
\hline \multirow[t]{4}{*}{ Age } & $17-20$ years & 2.94 & 2.04 & 2.07 & 0.15 & 18.83 & $0.35-1027.38$ \\
\hline & 21-24years & 0.96 & 1.64 & 0.34 & 0.56 & 2.61 & $0.11-64.91$ \\
\hline & 25-28years & 1.13 & 1.73 & 0.43 & 0.51 & 3.10 & $0.11-91.59$ \\
\hline & 29-32years & $0 \mathrm{~b}$ & & & & &. \\
\hline
\end{tabular}




\begin{tabular}{|l|l|l|l|l|l|l|l|}
\hline Gender & Female & 1.78 & 1.11 & 2.58 & 0.11 & 5.92 & $0.68-51.98$ \\
\hline & Male & $0 \mathrm{~b}$ &. &. &. &. \\
\hline Department & Medicine & 0.14 & 1.54 & 0.01 & 0.93 & 1.15 & $0.06-23.64$ \\
\hline & Nursing & -0.21 & 1.41 & 0.02 & 0.88 & 0.81 & $0.05-12.85$ \\
\hline & Physiotherapy & $0 \mathrm{~b}$ &. &. &. &. &.- \\
\hline Year in School & 3rd & -2.78 & 1.94 & 2.05 & 0.15 & 0.06 & $0.001-2.80$ \\
\hline & 4th & -1.83 & 1.59 & 1.32 & 0.25 & 0.16 & $0.007-3.64$ \\
\hline & 5th & -0.11 & 1.68 & 0.004 & 0.95 & 0.9 & $0.033-24.40$ \\
\hline & 6th & $0 \mathrm{~b}$ &. &. &. &. \\
\hline
\end{tabular}

The reference category is Low Stress (0-13); b This parameter is set to zero because it is redundant; * means significant at p< 0.05 PSS: Perceived Stress Scale; SE: Standard Error

Table 5b: Relationship between some demographic factors and perceived stress score after COVID-19 lockdown ( $\mathrm{n}=220)$

\begin{tabular}{|c|c|c|c|c|c|c|c|}
\hline Variables & Categories & B & SE & Wald & Sig. & $\operatorname{Exp}(B)$ & $\begin{array}{l}\text { 95\% CI for } \operatorname{Exp}(\mathrm{B}) \\
\text { Lower -Upper }\end{array}$ \\
\hline & Moderate Stress (14-26) & & & & & & \\
\hline & Intercept & 1.86 & 1.08 & 2.97 & 0.09 & & \\
\hline \multirow[t]{4}{*}{ Age } & $17-20$ years & 1.81 & 1.25 & 2.11 & 0.15 & 6.103 & $0.532-69.978$ \\
\hline & 21-24years & 1.83 & 1.09 & 2.80 & 0.09 & 6.209 & $0.731-52.729$ \\
\hline & 25-28years & 1.05 & 0.93 & 1.29 & 0.257 & 2.87 & $0.464-17.752$ \\
\hline & 29-32years & $0 \mathrm{~b}$ & & & & . &.. \\
\hline \multirow[t]{2}{*}{ Gender } & Female & -0.47 & 0.64 & 0.546 & 0.46 & 0.625 & $0.179-2.175$ \\
\hline & Male & $0 \mathrm{~b}$ & & & & & $\therefore$. \\
\hline \multirow[t]{3}{*}{ Department } & Medicine & -1.10 & 0.80 & 1.92 & 0.166 & 0.332 & $0.07-1.58$ \\
\hline & Nursing & 0.27 & 0.81 & 0.11 & 0.74 & 1.31 & $0.266-6.457$ \\
\hline & Physiotherapy & $0 \mathrm{~b}$ & & & & &. \\
\hline \multirow[t]{6}{*}{ Level } & $3 \mathrm{rd}$ & -0.35 & 1.38 & 0.065 & 0.799 & 0.705 & $0.047-10.457$ \\
\hline & 4th & -0.74 & 0.83 & 0.784 & 0.376 & 0.479 & $0.094-2.443$ \\
\hline & 5 th & -1.73 & 0.75 & 5.267 & $0.022 *$ & 0.177 & $0.04-0.777$ \\
\hline & 6th & $0 \mathrm{~b}$ & & & & & .-. \\
\hline & High Stress (27-40) & & & & & & \\
\hline & Intercept & 0.04 & 1.48 & 0.001 & 0.977 & & \\
\hline \multirow[t]{4}{*}{ Age } & $17-20$ years & 3.28 & 1.6 & 4.238 & $0.04 *$ & 26.66 & $1.171-607.172$ \\
\hline & 21-24years & 2.93 & 1.47 & 3.979 & $0.046^{*}$ & 18.809 & $1.053-336.074$ \\
\hline & 25-28years & 1.68 & 1.37 & 1.506 & $0.22 *$ & 5.367 & $0.367-78.544$ \\
\hline & 29-32years & $0 \mathrm{~b}$ & & & & &. \\
\hline \multirow[t]{2}{*}{ Gender } & Female & -0.95 & 0.72 & 1.742 & 0.187 & 0.389 & 0.095-1.582 \\
\hline & Male & $0 \mathrm{~b}$ & & & & &.$\therefore$ \\
\hline \multirow[t]{3}{*}{ Department } & Medicine & -1.09 & 0.90 & 1.466 & 0.226 & 0.335 & $0.057-1.966$ \\
\hline & Nursing & 1.15 & 0.86 & 1.7 & 0.192 & 3.081 & $0.568-16.72$ \\
\hline & Physiotherapy & $0 \mathrm{~b}$ & . & & . & . &..- \\
\hline \multirow[t]{4}{*}{ Year in School } & $3 \mathrm{rd}$ & -0.29 & 1.48 & 0.04 & 0.842 & 0.745 & $0.041-13.552$ \\
\hline & 4th & -0.86 & 1.00 & 0.742 & 0.389 & 0.422 & $0.059-3.003$ \\
\hline & 5 th & -2.08 & 0.93 & 4.959 & $0.026^{*}$ & 0.125 & $0.02-0.78$ \\
\hline & 6th & $0 \mathrm{~b}$ & & & & & $\therefore$ \\
\hline
\end{tabular}

The reference category is Low Stress (0-13); b This parameter is set to zero because it is redundant; * means significant at p< 0.05 PSS: Perceived Stress Scale; SE: Standard Error

Recommendations to university on how to help health sciences students cope with stress

Table 6 shows that the highest proportion of recommendations reported by the health sciences students was to reduce lecture load during online class $19.1 \%$, while $16.4 \%$ had no recommendation in case of another pandemic or COVID-19 lockdown.

\section{Discussion}

The literature has reported that the COVID-19 pandemic has significantly affected the mental health and occupation of the population worldwide [21, 22]. The prevalence of depression and anxiety has been reported to be up to $44 \%$ in developing countries, while a prevalence of $66.5 \%$ was reported in a survey done on health sciences students of Europe and the Englishspeaking regions outside North America [23, 24]. Stress, whether moderate or high, is very prevalent in health sciences students, which may not be unconnected to huge workload, long practical hours, and other academic stress, which pose a greater risk of physical and mental health issues to them than their counterparts in other fields [25, 26, 27]. 
Table 6: Recommendations to University on how to help Health Sciences Students cope with stress in case of another pandemic

\begin{tabular}{|l|l|}
\hline Recommendations & $\mathbf{N = 2 2 0 ( \% )}$ \\
\hline Reduce Frequency of Online Lectures & $42(19.1)$ \\
\hline Lockdown in School & $41(18.6)$ \\
\hline Awareness and Enlightenment of pandemic & $24(10.9)$ \\
\hline No Online Lectures & $22(10)$ \\
\hline Online Lectures preferred & $18(8.2)$ \\
\hline Provision of Data Subscriptions to students & $12(5.5)$ \\
\hline Counseling & $12(5.5)$ \\
\hline Psychotherapy & $7(3.2)$ \\
\hline $\begin{array}{l}\text { Less attention to news and updates related to } \\
\text { COVID-19 }\end{array}$ & $3(1.4)$ \\
\hline Encouragement and showing love to students & $2(0.9)$ \\
\hline No recommendation & $36(16.4)$ \\
\hline
\end{tabular}

In this study, most respondents have a high level of stress (46.8\%) during the COVID-19 lockdown and a moderate level of stress (58.2\%) after the COVID-19 resumption. Such finding is contrary to the study carried out by Abdulgani et al. [27] in Saudi Arabia, with only $12.8 \%$ of the respondents with high stress, and Deemah et al. [20], who indicated respondents had a moderate level of stress (55\%) during the COVID-19 lockdown. This difference in the level of stress could be due to the differences of the diagnostic instruments used. During the COVID-19 Lockdown, "how often did you feel upset because your studies changed unexpectedly" was the highest perceived stress among the respondents (Mean, $\mathrm{SD}=3.10,0.873$ ). Such a result may be due to the academic, financial, and social difficulties associated with online learning, as Kwaah and Essilfie [28] reported. Adjusting to the online learning model may be a challenge for these students as they have to learn new technology, insufficient home resources facilitating online learning, or unstable internet connection [29]. In addition, their frustration may also not be unassociated with the pressure to learn healthcare development skills online, which poses a serious challenge to these health sciences students [20, 30, 31]. During the covid-19 lockdown, the female participants demonstrated a moderate level of stress. Such finding is in line with the study of Torun and Torun [33]. Similarly, Bashir et al. [22] reported that women have more caregiving and household responsibilities which might have added to their stress. The participants in their 3rd year also demonstrated moderate stress during COVID-19 lockdown; this may be due to anxiety from compromised internet access or comprehension of online classes, as suggested by Bashir et al. [22].

Our study showed a significant relationship between the high level of perceived stress after COVID-19 resumption with participants of younger age (17-20 and 21-24 respectively). Our result is in concordance with studies conducted by Bashir et al. [22], Qin et al. [32], and Wang et al. [10], who also reported the younger age group to be associated with psychological symptoms of stress. A high level of stress may have been more pronounced in these age groups because of age-related hormonal disturbances, limitation of outdoor activities, and more access to social media-related disinformation [22].

Moderate and high-stress levels were observed in health sciences students of Bowen University in their 5th-year health sciences students after COVID-19 lockdown. The increase in stress level with a higher level of education status may be due to examination-related stress, compromised access and/or comprehension of online classes, and foreseeing delay in examination with the eventual outcome of challenges of job opportunities as suggested by Bashir et al. [22].

On the recommendations proffered by respondents in this study, most of them suggested that the lecture loads online be reduced (19.6\%), followed closely by $18.6 \%$, who suggested that the lockdown be observed in school. These recommendations may be connected to the boredom attached to sitting glued to the gadgets for several hours, disturbances and distractions from family and friends, erratic network, and cost implications attached to the internet. Those who preferred online lectures $(8.2 \%)$ were less than those who did not want online lectures (10\%), this may be due to the quest for interpersonal communication, which may be limited or lacking in online classes, as well as the cost implications attached to online classes with attending network challenges.

This study was conducted in just one private university and did not involve a public university. Also, the 600 level medicine students were writing exams in the course of this study; hence, the majority of them did not participate in this study. Recommendations and Future Studies. A replication study is recommended in another setting to promote the generalizability of the findings of this study. The University Management should also help to reduce the lecture load online in case of another pandemic. The research should be built upon; this may include and address additional variables to build on this research and study. The same research problems in this study can be addressed in a different setting, context, location in future studies.

\section{Implication to practice}

This study has identified the perceived stress during COVID-19 lockdown and after COVID-19 resumption among health science students in Bowen teaching hospital, which could lead to a significant health problem if not considered. The findings of this study are valuable to school counselors, college counselors, academic support personnel, and other higher education educators. A better understanding of the stress level in students will help the school authority lived to support the students more effectively. We aim for the implications of this study to spark consideration and innovation for preventative strategies and interventions that aim to help the students manage stress. Specifically, we hope university administrators strive to help the students cope with the stress associated with pandemics as recommended by the students.

\section{Conclusion}

This study identifies that health science students during the COVID-19 lockdown had a high level of stress and also identifies that after COVID-19 lockdown, health sciences students had moderate stress levels.

\section{Abbreviation}

COVID-19: The 2019 Corona Virus Disease; SARS-COV-2: Severe Acute Respiratory Syndrome Coronavirus-2; PSS: Perceived Stress Scale; IBM SPSS: International Business Machine Corporation Statistical Product and Service Solutions. 


\section{Declaration}

Acknowledgment

None.

\section{Funding}

The authors received no financial support for their research, authorship, and/or publication of this article.

Availability of data and materials

Data will be available by emailing peteradedeji2020@gmail.com

\section{Authors' contributions}

Peter Oluyemi Adedeji (POA) conceived the idea, contributed to the design of the questionnaire, wrote the ethical protocol and the manuscripts. Adenike Ayobola Olaogun (AAO) supervised the whole research, contributed to the design of the questionnaires, reviewed, and editing of the manuscripts. Marinella Precious Oyedeji (APO) created the google form and helped in data collection and editing the manuscript. Oluwatosin Adewusi Adedeji (OAA) spearheaded the analysis and editing of the manuscript. All authors have read and approved the final manuscript.

\section{Ethics approval and consent to participate}

We conducted the research following the declaration of Helsinki. The ethical protocol was approved by the Bowen University Teaching Hospital Research Ethics Committee (BUTHREC) with approval number BUTH/REC-147. Informed consent was also obtained from students before data collection through WhatsApp messages. Anonymity was maintained in the questionnaire as well.

Consent for publication

Not applicable

\section{Competing interest}

The authors declare that they have no competing interests.

Open Access

This article is distributed under the terms of the Creative Commons $\begin{array}{llll}\text { Attribution } & 4.0 & \text { International License }\end{array}$ (http://creativecommons.org/licenses/by/4.0/), which permits unrestricted use, distribution, and reproduction in any medium, provided you give appropriate credit to the original author(s) and the source, provide a link to the Creative Commons license, and indicate if changes were made. The Creative Commons Public Domain Dedication waiver (http://creativecommons.org/publicdomain/zero/1.0/) applies to the data made available in this article, unless otherwise stated.

\section{Author details}

${ }^{1}$ Department of Nursing Science, College of Health Sciences, Bowen University, Iwo, Osun State, Nigeria. ${ }^{2}$ Institute of Ecology \& Environmental Studies, Obafemi Awolowo University, Ile-Ife, Osun State, Nigeria.

\section{Article Info}

Received: 09 August 2021

Accepted: 23 September 2021

Published: 26 september 2021

\section{References}

1. Centers for Disease Control and Prevention (2020). Information for healthcare professionals about coronavirus (COVID-19)
[Internet]; 2020 [cited 2020 Jun 23]. https:// www.cdc.gov/coronavirus/2019-nCoV/hcp/index.html?CDC. [Accessed on 31st July 2020]

2. The National Health Commission (NHC) (2020). Daily announcements of the epidemic situation of China (April 18, 2020). National Health Commission (NHC) website. http://www.nhc.gov.cn/xcs/yqtb/202004/2d391a171acc4624a50a1 188c8de7361.shtml. [Accessed on August 6, 2020]

3. Ajisegiri WS, Odusanya OO and Joshi R. COVID-19 outbreak situation in Nigeria and the need for effective engagement of community health workers for epidemic response. Global Biosecurity. 2020;1(4). DOI: http://doi.org/10.31646/gbio.69

4. World Health Organisation (WHO) (2020). Coronavirus Disease (COVID-19) Events as they Happen; 2020. https://www.who.int/emergencies/diseases/novel-coronavirus2019/events-as-they-happen. [Accessed on September 21, 2020]

5. Aluko O. (2020). Coronavirus: FG orders closure of varsities, schools nationwide. The Punch Newspaper. (2020, March 19). Retrieved from HTTPS:// punchng.com/breaking-coronavirus-fgorders-closure-of-varsitiesschools-nationwide/

6. Ajayi O. (2020). Covid-19: FG shuts Enugu, Port Harcourt and Kano airports. Vanguard. (2020, March 21). Retrieved from http://www.vanguardngr.com/2020/03/covid-19-fg-shuts-enuguport-harcourt-and-kano-airports/

7. Abubakar S. (2020, March 20). Coronavirus: Osun bans public gatherings, shuts schools, religious centres. Vanguard. www. vanguardngr.com/2020/03/coronavirus-osun-bans-publicgatheringsshuts-schools-religious-centres/

8. Oginni OA, Amiola A, Adelola A, Uchendu U. A Commentary on the Nigerian response to the COVID-19 pandemic. Psychological Trauma: Theory, Research, Practice, and Policy.2020; June 11. Advance online publication. http://dx.doi.org/10.1037/tra0000743

9. Ogundele K (2020). Updated: FG places travel ban on China, Italy, U. S., U. K., nine others. Punch. 2020, March 18. Retrieved from https://punchng .com/breaking-fg-places-travel-ban-onchina-Italy-us-UK-others/

10. Wang C, Pan R, Wan X, Tan Y, Xu L, Ho CS, et al. Immediate Psychological Responses and Associated Factors during the Initial Stage of the 2019 Coronavirus Disease (COVID-19) Epidemic among the General Population in China. Int J Environ Res Public Health. 2020 Mar 6;17(5):1729.

11. The Academy of Mental Health Sciences. Survey results: Understanding people's concerns about the mental health impacts of the COVID-19 pandemic. 2020;(April). [Accessed on September 21, 2020]

12. Xiong J, Lipsitz O, Nasri F, Lui L, Gill H, Phan L, et al. Impact of COVID-19 pandemic on mental health in the general population: A systematic review. Journal of affective disorders 2020;277: 5564. https://doi.org/10.1016/j.jad.2020.08.001

13. Asad A, Srivastava S, Verma MK. Evolution of COVID-19 Pandemic in India. Transactions of the Indian National Academy of Engineering 2020; 1-8. Advance online publication. https://doi.org/10.1007/s41403-020-00166-y

14. Kakunje A, Mithur R and Kishor M (2020). Emotional well-being, mental health awareness, and prevention of suicide: Covid-19 pandemic and digital psychiatry. Arch Med Health Sci 2020;8:147-53

15. Wang J, Lloyd-Evans B, Giacco D, Forsyth R, Nebo C, Mann F, et al. Social isolation in mental health: A conceptual and 
methodological review. Soc Psychiatry Psychiatr Epidemiol 2017;52:1451-61.

16. Vala NH, Vachhani MV, Sorani AM. Study of anxiety, stress, and depression level among medical students during COVID-19 pandemic phase in Jamnagar city. Natl J Physiol Pharm Pharmacol 2020;10(12):1043-1045.

17. Basch CH, Hillyer GC, Meleo-Erwin ZC, Jaime C, Mohlman J, Basch CE. Preventive behaviours conveyed on YouTube to mitigate transmission of COVID-19: cross-sectional study. JMIR Public Heal Surveill. 2020 Apr 2;6(2):e18807.

18. Cuan-Baltazar JY, Muñoz-Perez MJ, Robledo-Vega C, PérezZepeda MF, Soto Vega E. COVID- 19 misinformation on the internet: the other epidemy (Preprint). JMIR Public Heal Surveill. 2020 Feb 26 [cited 2020 Apr 18];6(2):e18444.

19. Garrett L. COVID-19: the medium is the message. Lancet. 2020 March 21;395(10228):942-3. pmid:32171075

20. Deemah AA, Sumayah A, and Dalal A(2020). Perceived stress among students in virtual classrooms during the COVID-19 outbreak in KSA, Journal of Taibah University Medical Sciences.2020;15(5):398-403. https://doi.org/10.1016/j.jtumed.2020.07.004.

21. Ahmed MA, Jouhar R, Ahmed N, Adnan S, Aftab M, Zafar MS and Khurshid Z. Fear and practice modifications among dentists to combat novel coronavi $\neg$ rus disease (COVID-19) outbreak. Int J Environ Res Public Health 2020;17 (8):2821

22. Bashir T, Hassan S, Maqsood A, Khan ZA, Issrani R, Ahmed N, Bashir EF. The Psychological Impact Analysis of Novel COVID19 Pandemic in Health Sciences Students: A Global Survey. European journal of dentistry2020;14(S 01): S91-S96. https://doi.org/10.1055/s-0040-1721653

23. Rotenstein LS, Ramos MA, Torre M, Segal JB, Peluso MJ, Guille $\mathrm{C}$, Sen S and Mata DA. Prevalence of depres $\neg$ sion, depressive symptoms, and suicidal ideation among medical students: a systematic review and meta-analysis. JAMA 2016; 316(21):22142236.

24. Azad N, Shahid A, Abbas N, Shaheen A, Munir N. Anxiety and depression in medical students of a private medical college. J Ayub Med Coll Abbottabad 2017;29 (1):123-127
25. Saravanan C and Wilks R. Medical students' experience of and reaction to stress: the role of depression and anxiety. The Scientific World Journal, 2014, Article ID 737382. http://dx.doi.org/10.1155/2014/737382

26. Francis B, Gill JS, Yit Han N, Petrus CF, Azhar FL, Sabki ZA, Said MA, Hui KO, Guan NC and Sulaiman AH. Religious coping, religiosity, depression and anxiety among medical students in a multi-religious setting. Int $\mathrm{J}$ Environ Res Public Health. 2019;16:2.17.

27. Abdulghani HM, Sattar K, Ahmad T, and Akram A. Association of COVID-19 pandemic with undergraduate medical students' perceived stress and coping. Psychol Res Behav Manag. 2020;13: 871-881. https://doi.org/10.2147/PRBM.S276938

28. Kwaah CY and Essilfie G. Stress and coping strategies among distance education students at the University of Cape Coast, Ghana. Turk Online J Dist Educ 2017; 18(3): 120e134.

29. Sahu P. Closure of universities due to coronavirus disease 2019 (COVID-19): impact on education and mental health of students and academic staff. Cureus [Internet] 2020 Apr 4; 12(4):e7541. Available from: https://pubmed.ncbi.nlm.nih.gov/32377489.

30. Triola MM, Friedman E, Cimino C, Geyer EM, Wiederhorn J, Mainiero C. Health information technology and the medical school curriculum. Am J Manag Care. 2010;16(12 Suppl HIT):Sp54-Sp56.30.

31. Thompson P. The digital natives as learners: Technology use patterns and approaches to learning. Comput Educ. 2013; 65:1233. doi:10.1016/j.compedu.2012.12.022

32. Qin J, Shen B, Zhao M, Wang Z, Xie B, Xu Y . A nationwide survey of psychological distress among Chinese people in the COVID-19 epidemic: implications and policy rec-ommendations. General Psychiatry 2020;33(2)10.1136/ gpsych-2020-100213.

33. Torun F, Torun SD. The psychological impact of the COVID-19 pandemic on medical students in Turkey. Pakistan journal of medical sciences2020;36(6): 1355-1359. https://doi.org/10.12669/pjms.36.6.298510.1055/s-0040-1710050. 